\title{
Descubriendo el valor al cliente en empresas de servicios desde un modelo de aprendizaje basado en problemas
}

\section{Discovering customer value in services companies from a problem-based learning model}

ÁNGEL F. VILLAREJO-RAMOS

ORCID: https://orcid.org/0000-0002-6916-2839

Universidad de Sevilla.

Departamento de Administración

de Empresas y Marketing

curro@us.es

Fecha de recepción:

Fecha de aceptación:

DOI: http://dx.doi.org/10.12795/9788447221912.073

Pp.: 1678-1704 


\section{Resumen}

Presentamos el ciclo de mejora en el aula (CIMA) diseñado y aplicado para la asignatura de Marketing de Servicios en el Grado en Marketing e Investigación de Mercados de la Universidad de Sevilla. El tema elegido para su aplicación ha sido en de la gestión del valor al cliente. El modelo metodológico aplicado pretende dar un papel más protagonista al estudiante, basado el aprendizaje en el planteamiento de problemas a resolver para ir alcanzando por si mismos los conocimientos teóricos que componen los contenidos conceptuales del programa. Tras la aplicación del mismo logramos una evaluación satisfactoria tanto en la mejora del proceso de aprendizaje a nivel grupal e individual, así como una evaluación favorable de la mejora lograda en la labor del docente como guía más que transmisor del conocimiento en el proceso de enseñanza de la materia.

Palabras clave: docencia universitaria, marketing de servicios, grado en marketing, aprendizaje basado en problemas, experimentación docente universitaria

\section{Abstract}

We present the improvement cycle in classroom (ICIC) designed and applied to the subject of Services Marketing in the Degree in Marketing and Market Research at the University of Sevilla. The topic chosen for its application has been in the management of customer value. The methodological model applied aims to give a more prominent role to the student, based on learning the approach of problems to solve to reach by themselves the theoretical knowledge that make up the conceptual content of the programme. After the application of the same one we obtained a satisfactory evaluation so much in the improvement of the process of learning to group and individual level, as well as a favourable evaluation of the improvement obtained in the work of the teacher like guide more than transmitter of the knowledge in the process of teaching of the matter.

Keywords: university teaching, services marketing, marketing degree, problem-based learning, university teaching experimentation 


\section{Contexto de aplicación del ciclo de mejora en el aula (CMA)}

La experiencia para mejorar la docencia a través de la aplicación de un clico de mejora en el aula (CIMA), la hemos realizado para la asignatura de Marketing de Servicios, materia obligatoria para los estudiantes del único grupo del cuarto curso del Grado en Marketing e Investigación de Mercados de la Universidad de Sevilla. El número de alumnos matriculados en la asignatura que optan por el sistema de evaluación continua es muy elevado, más de 80, si bien la participación en el CIMA de una forma activa, con asistencia y participación en todas las sesiones, ha sido considerablemente menor, contando con 46 observaciones completas que nos permitirán evaluar el proceso de enseñanza-aprendizaje. Además del numeroso grupo, el tamaño del aula, con capacidad para más de 150 estudiantes y la disposición en teatro con asientos fijos, han dificultado el correcto desarrollo del trabajo en grupos, pieza central de las actividades planteadas en el modelo. Creemos también relevante señalar que la docencia en este grupo es compartida, por lo que los estudiantes han recibido docencia de los dos temas anteriores a la aplicación del CIMA con otro profesor y siguiendo la metodología usada en cursos anteriores, en las que las sesiones teóricas y prácticas no siguen el modelo metodológico que hemos utilizado en esta experimentación.

\section{Diseño previo del CIMA}

El inicio del CIMA venía precedido del contacto con los estudiantes del grupo en la semana anterior. Pedimos a nuestro compañero de asignatura que nos cediera media hora de su clase. En este tiempo, planteamos la situación del contexto inicial en la que los estudiantes imaginaban ser director/a de marketing de MOVING'1 empresa dedicada al alquiler de motocicletas urbanas eléctricas. Ante el 
problema detectado en la caída de los ingresos de la empresa en el segundo cuatrimestre del año 2019, el director/a de marketing se plantea una serie de preguntas a las que quiere dar respuesta previa a la reunión que mantendrá en los próximos días con su equipo. Ante esta situación y para dar respuesta al problema de la caída de los ingresos, definimos los dos problemas a resolver: P1 ¿Por qué está perdiendo clientes la empresa MOVING? y P2 ¿Cómo podemos mejorar el valor al cliente en la empresa MOVING? (ver figura 1). Descomponemos estos dos problemas en cuatro preguntas planteadas, dos para cada problema, que conformaron el cuestionario inicial y final.

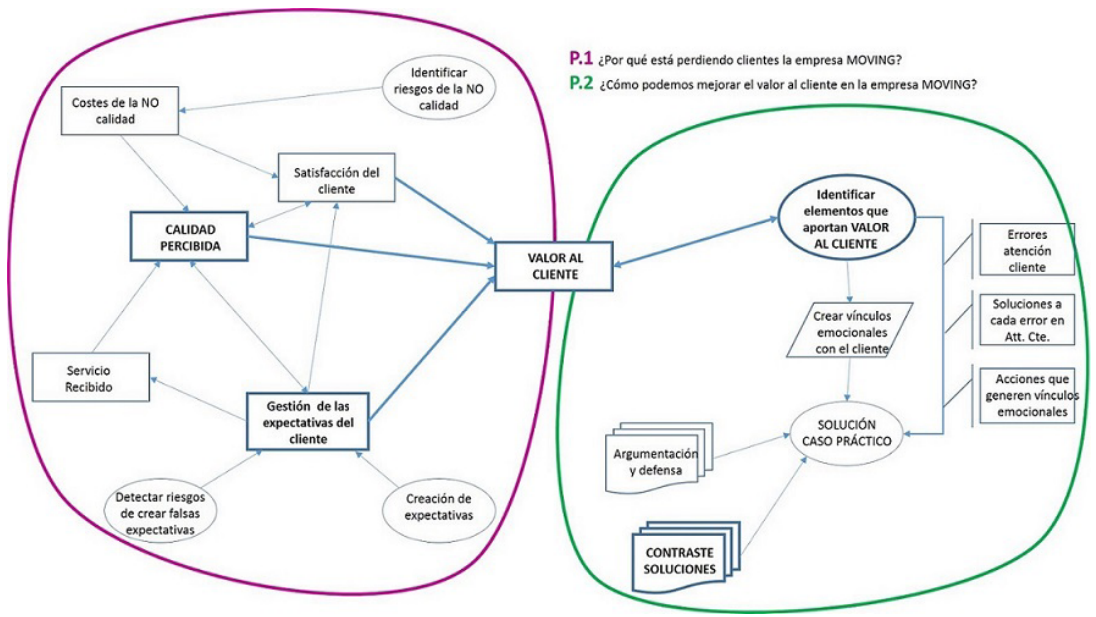

Figura 1: Mapa de contenidos y problemas

Leyenda Mapa de Contenidos: En negrita y mayor grosor de línea: contenidos estructuradores

Contenidos conceptuales $\square$ Contenidos procedimentales
Contenidos actitudinales. $\square$ Procedimientos intelectuales

En el modelo metodológico posible hemos dado un papel protagonista al estudiante, la educación centrada en el estudiante más que en la disciplina impartir o en el docente (Bain, 2007). Hacemos al alumno más partícipe del proceso de enseñanza-aprendizaje al plantearle problemas encadenados que debe ir resolviendo a través de reflexión individual y consenso en equipos de trabajo de 4-5 
compañeros. El docente asume un papel más de guía y con una presencia más de apoyo que de transmisor de los conocimientos (Finkel, 2008). Pretendemos trasladar a la docencia un modelo basado en una metodología basada en la atención captada en el alumnado, suscitando su curiosidad y el interés por la investigación, para conseguir un aprendizaje duradero en el tiempo (Mora, 2017).

En la figura 2 se detalla el modelo metodológico propuesto en el que se observa la importancia dada al trabajo de los estudiantes, tanto a nivel individual como grupal. Asimismo, destacamos el modelo cíclico alrededor de los problemas que se van abordando con preguntas encadenadas a lo largo del ciclo completo. La secuencia de actividades para cada una de las cuatro sesiones en las que se ha aplicado el CIMA se detallan en la tabla 1.

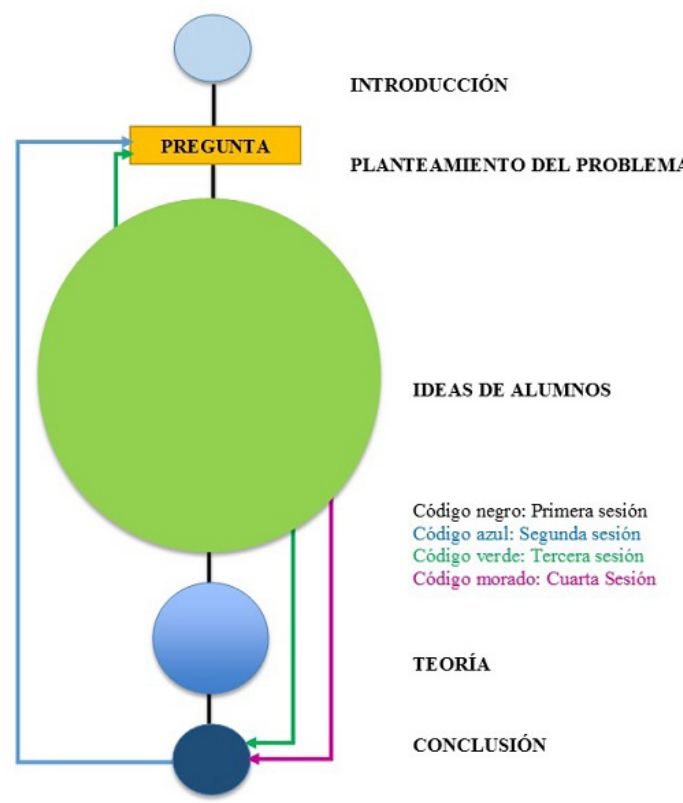

Figura 2: Modelo metodológico

Jornadas de Formación e Innovación Docente del Profesorado | № 2 (2019) Esta obra se distribuye con la licencia Creative Commons 
Tabla 1: Secuencia de actividades

\begin{tabular}{|c|c|c|c|}
\hline \multicolumn{4}{|c|}{ Sesión 1: ¿Por qué está perdiendo clientes la empresa MOVING? I de II } \\
\hline Actividad & & Descripción & Tiempo \\
\hline \multirow[t]{2}{*}{ Introducción } & I.1 & $\begin{array}{l}\text { Introducción por parte del profesor sobre el } \\
\text { CASO MOVING y la situación detectada tras los } \\
\text { informes de ventas y sobre las descargas de } \\
\text { apps de MOVING. }\end{array}$ & $5 \mathrm{~min}$. \\
\hline & 1.2 & $\begin{array}{l}\text { Los equipos de trabajo de la asignatura } \\
\text { (cuatro-cinco alumnos) deben sentarse en } \\
\text { círculo y ocupando todo el espacio del aula. } \\
\text { Cada grupo se autodenominará con el } \\
\text { nombre de alguna marca de servicios que } \\
\text { los identifique, salvo la marca protagonista } \\
\text { del caso. Entregaremos a los grupos la ficha } \\
\text { del caso en la que aparecerá el nombre del } \\
\text { grupo, sus componentes y espacio para ir } \\
\text { completando en las cuatro sesiones. }\end{array}$ & $5 \mathrm{~min}$. \\
\hline $\begin{array}{l}\text { Planteamiento } \\
\text { del Problema }\end{array}$ & P.1.1 & $\begin{array}{l}\text { Se inicia con el resumen del profesor sobre las } \\
\text { respuestas dadas a la primera pregunta del } \\
\text { cuestionario inicial. } \\
\text { ¿Cuáles pueden ser los motivos por los que } \\
\text { clientes y usuarios actuales de MOVING, } \\
\text { han dejado de utilizar el servicio en este } \\
\text { cuatrimestre? }\end{array}$ & $10 \mathrm{~min}$. \\
\hline \multirow[t]{2}{*}{$\begin{array}{l}\text { Ideas de } \\
\text { alumnos }\end{array}$} & IA.1 & $\begin{array}{l}\text { Los alumnos debatirán en sus respectivos } \\
\text { equipos sobre los motivos que consideran } \\
\text { han podido causar la caída de las ventas } \\
\text { provocadas por el descenso en la tasa de uso } \\
\text { de los clientes. } \\
\text { Tomarán nota en la ficha del caso de cada } \\
\text { equipo de todas las ideas principales a las } \\
\text { que lleguen por consenso y que tendrán } \\
\text { que ponderar en orden de importancia. } \\
\text { La selección final será de cinco motivos } \\
\text { principales. }\end{array}$ & $35 \mathrm{~min}$. \\
\hline & IA.2 & $\begin{array}{l}\text { Se eligen tres de los equipos. Sus portavoces } \\
\text { escribirán en la pizarra, dividida en tres partes, } \\
\text { los cinco motivos priorizados por su equipo. }\end{array}$ & $25 \mathrm{~min}$ \\
\hline
\end{tabular}

Jornadas de Formación e Innovación Docente del Profesorado | № 2 (2019)

Esta obra se distribuye con la licencia Creative Commons

Reconocimiento-NoComercial-SinObraDerivada

Internacional (CC BY-NC-ND 4.0.) 


\begin{tabular}{|c|c|c|c|}
\hline Teoría & T.1 & $\begin{array}{l}\text { El profesor tratará de enlazar las ideas de } \\
\text { los estudiantes con el desarrollo de dos } \\
\text { conceptos principales: costes de la NO calidad } \\
\text { y gestión de las expectativas de los clientes. } \\
\text { La explicación del profesor será ilustrada con } \\
\text { ejemplos y apoyo de una presentación de } \\
\text { tres-cinco diapositivas (material que estará } \\
\text { disponible para estudiantes en EV al final del } \\
\text { tema). }\end{array}$ & $20 \mathrm{~min}$. \\
\hline Conclusión & C.1 & $\begin{array}{l}\text { A continuación, el profesor lanza una nueva } \\
\text { pregunta para la reflexión y para seguir } \\
\text { avanzando en el intento de encontrar } \\
\text { explicaciones a la caída de ventas de MOVING. } \\
\text { ¿Por qué han bajado su nivel de uso del } \\
\text { servicio los clientes MOVING actuales? }\end{array}$ & $10 \mathrm{~min}$. \\
\hline \multicolumn{4}{|c|}{ Sesión 2: ¿Por qué está perdiendo clientes la empresa MOVING? II de II } \\
\hline Actividad & & Descripción & Tiempo \\
\hline $\begin{array}{l}\text { Planteamiento } \\
\text { del problema }\end{array}$ & P.1.2 & $\begin{array}{l}\text { Se inicia con el resumen del profesor sobre las } \\
\text { respuestas dadas a la segunda pregunta del } \\
\text { cuestionario inicial. Se retoma } \\
\text { la pregunta de la sesión anterior para la } \\
\text { reflexión: } \\
\text { ¿Por qué han bajado su nivel de uso del } \\
\text { servicio los clientes de MOVING actuales? }\end{array}$ & $10 \mathrm{~min}$. \\
\hline \multirow[t]{2}{*}{$\begin{array}{l}\text { Ideas de } \\
\text { alumnos }\end{array}$} & IA.3 & $\begin{array}{l}\text { Se reúnen de nuevo los mismos equipos } \\
\text { y tratan de dar respuesta a la pregunta. } \\
\text { Sus causas pueden coincidir o no con las } \\
\text { planteadas inicialmente. Lo importante es } \\
\text { que el grupo vuelva a estar de acuerdo en las } \\
\text { causas que dejará por escrito la ficha del caso. }\end{array}$ & $35 \mathrm{~min}$. \\
\hline & IA.4 & $\begin{array}{l}\text { Se eligen dos nuevos grupos diferentes de } \\
\text { la sesión anterior que explica, a través de su } \\
\text { portavoz, lo que su grupo respectivo considera } \\
\text { las tres causas principales que ha provocado } \\
\text { que los usuarios de MOVING usen menos } \\
\text { el servicio de alquiler de motocicletas. Se } \\
\text { insta a que la clase plantee soluciones a los } \\
\text { problemas que llevamos detectados. }\end{array}$ & $25 \mathrm{~min}$. \\
\hline
\end{tabular}




\begin{tabular}{|c|c|c|c|}
\hline Teoría & T.2 & $\begin{array}{l}\text { Explicación de los conceptos de satisfacción } \\
\text { del cliente y calidad percibida. Junto a los } \\
\text { conceptos aprendidos de costes de no } \\
\text { calidad y gestión de las expectativas, nos } \\
\text { aproximamos al concepto de VALOR PARA EL } \\
\text { CLIENTE. } \\
\text { La explicación del profesor será ilustrada con } \\
\text { ejemplos y apoyo de una presentación de } \\
\text { tres-cinco diapositivas (material que estará } \\
\text { disponible para estudiantes en EV al finalizar } \\
\text { el tema). }\end{array}$ & $25 \mathrm{~min}$. \\
\hline Conclusión & C. 2 & $\begin{array}{l}\text { A continuación, el profesor lanza una nueva } \\
\text { pregunta para la reflexión y para seguir } \\
\text { avanzando en el intento de encontrar } \\
\text { explicaciones a la caída de ventas de MOVING. } \\
\text { ¿Cómo podemos mejorar el valor al cliente en } \\
\text { la empresa MOVING? }\end{array}$ & $10 \mathrm{~min}$. \\
\hline \multicolumn{4}{|c|}{ Sesión 3: ¿Cómo podemos mejorar el valor al cliente en la empresa MOVING? I de II } \\
\hline Actividad & & Descripción & Tiempo \\
\hline $\begin{array}{l}\text { Planteamiento } \\
\text { del problema }\end{array}$ & P.2 & $\begin{array}{l}\text { Se inicia la sesión retomando la pregunta } \\
\text { lanzada en la sesión anterior para la reflexión: } \\
\text { ¿Cómo podemos mejorar el valor al cliente en } \\
\text { la empresa MOVING? }\end{array}$ & $5 \mathrm{~min}$ \\
\hline \multirow[t]{3}{*}{$\begin{array}{l}\text { Ideas de } \\
\text { Alumnos }\end{array}$} & IA.5 & $\begin{array}{l}\text { Brainstorming dando respuesta a la cuestión } \\
\text { planteada como conclusión de la sesión } \\
\text { anterior. Cada alumno que interviene aporta } \\
\text { una idea que va recogiendo el profesor sobre } \\
\text { la pizarra. }\end{array}$ & $10 \mathrm{~min}$. \\
\hline & P.2.1 & $\begin{array}{l}\text { Se inicia con el resumen del profesor sobre } \\
\text { las respuestas dadas a la tercera pregunta del } \\
\text { cuestionario inicial. } \\
\text { ¿Qué podemos hacer para que nuestros } \\
\text { clientes actuales MOVING utilicen más el } \\
\text { servicio? } \\
\text { Encontrar ideas para incrementar el nivel de } \\
\text { uso del servicio. }\end{array}$ & $10 \mathrm{~min}$ \\
\hline & IA.6 & $\begin{array}{l}\text { Se reúnen de nuevo los mismos equipos con } \\
\text { sus fichas de sesiones anteriores y tratan de } \\
\text { dar respuesta a la pregunta. Lo importante } \\
\text { es que, de nuevo, el grupo vuelva a estar de } \\
\text { acuerdo en las ideas que dejará por escrito y } \\
\text { que, como siempre, irán ordenadas por orden } \\
\text { de importancia. Breve debate de ideas. }\end{array}$ & $35 \mathrm{~min}$. \\
\hline
\end{tabular}




\begin{tabular}{|c|c|c|c|}
\hline & P.2.2 & $\begin{array}{l}\text { Se inicia con el resumen del profesor sobre } \\
\text { las respuestas dadas a la cuarta pregunta del } \\
\text { cuestionario inicial. } \\
\text { ¿Qué acciones podemos llevar a cabo para } \\
\text { que los clientes de MOVING vean en nuestro } \\
\text { servicio una solución sostenible a la movilidad } \\
\text { urbana? }\end{array}$ & $10 \mathrm{~min}$. \\
\hline & IA.7 & $\begin{array}{l}\text { Los mismos equipos vuelven a reunirse para } \\
\text { enunciar acciones y consensuar sus ideas. } \\
\text { Todo se quedará por escrito en la ficha del } \\
\text { caso. Breve debate de ideas, destacando las } \\
\text { acciones más originales entre las planteadas. }\end{array}$ & $35 \mathrm{~min}$. \\
\hline Conclusión & C.3 & $\begin{array}{l}\text { A continuación, el profesor lanza quinta } \\
\text { pregunta para la reflexión y para seguir } \\
\text { avanzando en el intento de encontrar } \\
\text { soluciones para MOVING. } \\
\text { ¿Cómo podemos cuidar la relación emocional/ } \\
\text { afectiva con el cliente de MOVING? }\end{array}$ & $5 \mathrm{~min}$. \\
\hline \multicolumn{4}{|c|}{ Sesión 4: ¿Cómo podemos mejorar el valor al cliente en la empresa MOVING? II de II } \\
\hline Actividad & & Descripción & Tiempo \\
\hline $\begin{array}{l}\text { Planteamiento } \\
\text { del problema }\end{array}$ & P.2.3 & $\begin{array}{l}\text { Se inicia la sesión retomando la pregunta } \\
\text { lanzada en la sesión anterior para la reflexión: } \\
\text { ¿Cómo podemos cuidar la relación emocional/ } \\
\text { afectiva con el cliente de MOVING? }\end{array}$ & $5 \mathrm{~min}$. \\
\hline $\begin{array}{l}\text { Ideas de } \\
\text { Alumnos }\end{array}$ & IA.8 & $\begin{array}{l}\text { Los grupos de estudiantes van a repasar su } \\
\text { ficha del caso con la respuestas a las cuatro } \\
\text { preguntas, y van a intentar encontrar: } \\
\text { 1) Errores que se ha podido cometer MOVING } \\
\text { con respecto a la atención al cliente y que han } \\
\text { restado valor para el mismo; } 2 \text { ) Soluciones a } \\
\text { esos errores; y 3) Propuesta de acciones para } \\
\text { generar vínculo emocional con el cliente } \\
\text { Estas propuestas (3-5), consensuadas, y } \\
\text { ordenadas por importancia, deben recogerse } \\
\text { en la ficha del caso para cada grupo. }\end{array}$ & $40 \mathrm{~min}$. \\
\hline Teoría & AC.1 & $\begin{array}{l}\text { Dos grupos elegidos presentarán sus } \\
\text { propuestas a la clase y otros dos grupos } \\
\text { elegidos actuaran como relatores de las } \\
\text { propuestas de los grupos anteriores (cada } \\
\text { grupo expositor con su grupo relator). }\end{array}$ & $25 \mathrm{~min}$. \\
\hline
\end{tabular}




\begin{tabular}{|l|l|l|l|}
\hline & T.3 & $\begin{array}{l}\text { El profesor va guiando el debate destacando } \\
\text { los conceptos teóricos que aparecen en la } \\
\text { discusión, tratando de generalizarlo para otros } \\
\text { contextos diferentes. }\end{array}$ & 15 min. \\
\hline Conclusión & C.4 & $\begin{array}{l}\text { Se reparte el cuestionario final para que los } \\
\text { alumnos respondan de nuevo a las mismas } \\
\text { preguntas que en el cuestionario inicial. } \\
\text { Se recogen cuestionarios y las fichas de los } \\
\text { grupos. En la siguiente sesión se hará la } \\
\text { evaluación. }\end{array}$ & 30 min. \\
\hline
\end{tabular}

\section{Aplicación del CIMA}

\section{Diario de las sesiones}

Sesión 1 (martes, 29 de octubre de 2019). Al inicio de la clase recordamos, brevemente, el contexto inicial que planteamos la semana anterior y que nos serviría para la explicación del tema 3 que iniciamos este día. Insté a los estudiantes a formar grupos de cuatro-cinco personas y que se ubicasen por todo el espacio de la gran aula que tenemos. Formamos doce equipos de 4-5 estudiantes que se sentaron en forma circular (2 delante y dos detrás) y se esparcieron por todo el espacio disponible. En estos momentos, noté que no contaba con las mejores condiciones en cuanto al espacio de trabajo ni al número de estudiantes en la clase (más de 50).

Explicamos que para trabajar en las cuatro sesiones utilizaremos una ficha del caso que iremos completando con el trabajo que realizaremos en las cuatro sesiones del ciclo.

Antes de iniciar la actividad de trabajo de los estudiantes, los siguientes minutos se dedicaron al planteamiento del primero de los problemas: P.1.1. ¿Cuáles pueden ser los motivos por los que clientes y usuarios actuales de MOVING, han dejado de utilizar el servicio en este 
cuatrimestre? Con la información proveniente del análisis de las respuestas del cuestionario inicial hicimos un breve resumen de la escalera planteada con tres niveles de modelos de respuestas, que situaban en el nivel inferior al $17,4 \%$ de los individuos, con un reparto igual de respuestas en los otros dos niveles superiores.

Tras esto, los estudiantes iniciaron su reflexión por equipos para tratar de encontrar los cinco motivos principales, ordenados por importancia según el grupo, por los que los clientes actuales de MOVING han dejado de utilizar el servicio en el pasado cuatrimestre. Noto interés por participar dentro del grupo a la mayoría de los estudiantes y creo que están motivados para tratar de dar respuestas adecuadas a la pregunta planteada. Durante el tiempo que dura esta actividad el profesor recorre la clase y se muestra interesado por lo que están trabajando y accesible por si tienen cualquier duda o aclaración que precisen de él.

Al terminar el tiempo de trabajo de esta actividad se piden tres equipos que mediante un portavoz nos quieran explicar y mostrar sus motivos en la pizarra. Se ofrecen tres grupos, y entre ellos eligen a su portavoz. Los tres portavoces van explicando alternativamente cada uno de sus motivos expuestos por orden de importancia y los van recogiendo en la pizarra que previamente se ha dividido en tres y encabezado con el nombre de cada grupo de los voluntarios. La labor de los portavoces fue bastante dinámica, explicando con claridad sus motivos elegidos como causas que explicaban la no utilización del servicio por parte de los clientes. Algunos estudiantes desde la bancada y yo mismo intervenimos en alguna ocasión para aclarar alguna idea.

Terminada la intervención de los portavoces, con la pizarra llena de ideas, se fueron extrayendo los conceptos claves que surgen de estas ideas y que forman el contenido conceptual de la materia. En la explicación de los conceptos estuvieron muy participativos los estudiantes de los otros grupos que no habían sido voluntarios. Concluimos la sesión lanzando para la reflexión, de cara a la 
siguiente sesión, la segunda de las preguntas P.1.2. ¿Por qué han bajado su nivel de uso del servicio los clientes MOVING actuales?

Sesión 2 (jueves, 31 de octubre de 2019). La segunda sesión la iniciamos con el resumen de la observación y análisis de las respuestas dadas por estudiantes en el cuestionario inicial a la segunda de las preguntas. En esta sesión se produjo una reducción del número de estudiantes con lo que los grupos no se mantuvieron respecto al día anterior, y por ello, se dificultó el trabajo y la posibilidad de enlazar las repuestas dadas en la sesión 1.

Aun así y pese a todo mantuvimos la metodología propuesta, los equipos que se mantuvieron y los que se tuvieron que rehacer, trabajaron el segundo de los problemas que se habian planteado y recogieron en las fichas del caso, las que consideraron las tres causas principales por las que los clientes han bajado su tasa de utilización del servicio en el cuatrimestre pasado.

Transcurrido el tiempo de trabajo de los grupos, en los que el profesor volvió a estar accesible y recorriendo, de vez en cuando el aula, instamos a que salieran tres nuevos grupos voluntarios con su portavoz. La ausencia de grupos voluntarios obligó a elegir al azar a tres grupos que no fuesen los mismos que salieron voluntarios en la sesión 1. La intervención de los portavoces de los nuevos grupos estuvo bastante bien y nos permitió enlazar esas respuestas que dejaron sobre la pizarra con la explicación de los conceptos centrales previstos para este día en la actividad de explicación teórica.

El profesor realiza la explicación relacionando las causas que habían detectado en las respuestas sobre la pizarra con los conceptos teóricos que nos llevan a entender el concepto central del tema, el valor al cliente. La presentación en Power Point que había preparado el profesor sirvió para mostrar la relación de los conceptos de la sesión anterior con el concepto central de valor al cliente. 
La presentación final del tema la tuvieron disponible en la enseñanza virtual después de esta sesión.

Concluimos la sesión con el planteamiento del segundo gran problema a resolver a la empresa MOVING: ¿Cómo podemos mejorar el valor al cliente en la empresa MOVING?

Sesión 3 (lunes, 4 de noviembre de 2019). La sesión se inició con un brainstorming, dirigido por el docente, para dar ideas que resolviesen el segundo problema planteado que fue enunciado en la sesión 2 y que recogía la importancia de generar valor al cliente como núcleo conceptual del tema. ¿Cómo podemos mejorar el valor al cliente en la empresa MOVING? Las ideas planteadas a nivel individual por alumnos que participaron voluntariamente fueron recogidas en la pizarra.

Tras recoger y debatir sobre estas ideas la sesión estaba programada para acometer la solución a las dos preguntas planteadas del segundo problema. Esta solución se haría en grupos (formamos como pudimos los grupos con los alumnos presentes) y se recogería en las fichas del caso. Primero enunciamos la P.2.1. ¿Qué podemos hacer para que nuestros clientes actuales MOVING utilicen más el servicio? Que trabajaron durante 35 minutos. A continuación se enuncia la P.2.2. ¿Qué acciones podemos llevar a cabo para que los clientes de MOVING vean en nuestro servicio una solución sostenible a la movilidad urbana? Y se vuelve a trabajar en grupos durante otros 35 minutos. Durante este tiempo de trabajo por parte de los grupos de estudiantes, volvió el profesor a estar disponible y recorriendo el aula para ver el trabajo que estaban realizando los grupos y cómo los estaban sintetizando en las fichas del caso.

Algunas de las ideas de esta última pregunta fueron recogidas en la pizarra por parte del profesor, pidiendo a cada uno de los grupos que manifestasen sus acciones principales señaladas. Con estas ideas pudimos enlazar con la reflexión final que enlazaba los conceptos de valor 
al cliente con aspectos emocionales/afectivos para influir en la relación de MOVING con sus clientes. La conclusión del profesor instaba a reflexionar de cara a la siguiente sesión sobre la última de las preguntas planteadas para resolver el problema de la gestión del valor al cliente, que era: ¿Cómo podemos cuidar la relación emocional/afectiva con el cliente de MOVING?

Entre la sesión tercera y cuarta, ante la caída de alumnos en las sesiones, el profesor decide enviar mensaje a través de la plataforma de enseñanza virtual a todos los estudiantes del grupo, para hacer ver la importancia de la participación regular de todos en este modelo de aprendizaje y que necesitamos conocer la opinión final, a través del cuestionario final que se pasará en clase, para poder realizar la evaluación del tema.

Sesión 4 (jueves, 7 de noviembre de 2019). En la última sesión y por el efecto comunicación entre sesiones tuvimos, de nuevo, un elevado número de estudiantes. Al inicio de la sesión, a modo de introducción, se explicó el sentido que ha tenido la implantación de este modelo de aprendizaje y reconocemos el error de no haber motivado más a la participación y a la necesidad de la consistencia de los grupos durante todo el proceso, en aras a poder evaluar el trabajo realizado por cada estudiante y por los grupos constituidos.

Continúa la clase, descomponiendo la pregunta P.2.3., lanzada al final de la sesión anterior, en tres aparatados que le servirán para su trabajo en grupo y para recoger sus propuestas finales en la ficha del caso. Esta descomposición es: 1) Errores que ha podido cometer MOVING con respecto a la atención al cliente y que han restado valor para el mismo; 2) Soluciones a esos errores; y 3) Propuesta de acciones para generar vínculo emocional con el cliente.

Se les pide que trabajen sobre ello durante 40 minutos y que traten de recoger al final, tres-cinco propuestas concretas de acciones para generar mayor valor al cliente de MOVING. Tras el trabajo grupal propongo que dos grupos salgan a exponer sus propuestas (punto tres) que serán 
rebatidas y criticadas por otros dos grupos que actuarán como relatores de las propuestas presentadas. Este modelo de ponente-relator tuvo cierto éxito y en una de las parejas de grupos, se abrió un debate interesante entre las opiniones de uno y otro grupo.

Concluimos, tras el debate, con las ideas principales que nos han llevado a hacer propuestas concretas a la empresa para solucionar sus problemas de caída de los ingresos por ventas. Esto lo hemos hecho desde el análisis de las posibles causas y motivos que han podido estar en el origen de esa pérdida de la confianza por parte de los clientes.

Tras esta conclusión se pide a los diferentes grupos que entreguen las fichas del caso que hemos venido trabajando en las sesiones. Solo algunos de los grupos han mantenido una mínima consistencia en las cuatro sesiones, los demás han tenido días de más o menos participación y cambios en los componentes de los grupos, lo que me dificultará, enormemente, la posible evaluación grupal de la enseñanza.

Antes de finalizar la sesión, en lo últimos 25 minutos, se entrega el cuestionario final para que lo cumplimenten, poniendo el código individual y su nombre y apellidos para poder unir este cuestionario con el cuestionario inicial cumplimentado antes de la aplicación del CIMA. Como era de esperar muchos de los presentes no habían realizado el cuestionario inicial (tenía 46 cuestionarios iniciales y había 65 alumnos en el aula), por lo que a estos se les pide que lo cumplimenten y pongan su nombre.

\section{Evaluación del aprendizaje de los estudiantes}

El cuestionario para la evaluación del aprendizaje de los estudiantes fue diseñado para desarrollar los dos problemas planteados en el mapa de contenidos para la aplicación del CIMA. Cada uno de estos problemas fue descompuesto en dos preguntas que conformaban el cuestionario inicial de 4 preguntas escalonadas que fueron utilizadas en las sesiones de aplicación del ciclo de mejora del aprendizaje. 
El contexto situacional presentado para el desarrollo del CIMA situaba al estudiante en el papel de director/a de marketing de una empresa de servicios que venía detectando una caída de las ventas de su servicio provocada por la bajada de la tasa de utilización de clientes actuales y la menor incorporación de nuevos clientes. Así, planteamos un primer problema (P.1) enunciado como: ¿Por qué está perdiendo clientes la empresa MOVING? Este primer problema se descompuso en dos preguntas encadenadas que constituyeron las dos primeras preguntas del cuestionario: P.1.1 ¿Cuáles pueden ser los motivos por los que clientes y usuarios actuales de MOVING, han dejado de utilizar el servicio en este cuatrimestre? Y P.1.2 ¿Por qué han bajado su nivel de uso del servicio los clientes MOVING actuales?

El segundo de los problemas (P.2) se enunció con el siguiente texto: ¿Cómo podemos mejorar el valor al cliente en la empresa MOVING? En el cuestionario se plantearon para su solución dos preguntas: P.2.1 ¿Qué podemos hacer para que nuestros clientes actuales MOVING utilicen más el servicio? Y P.2.2 ¿Qué acciones podemos llevar a cabo para que los clientes de MOVING vean en nuestro servicio una solución sostenible a la movilidad urbana?

El cuestionario inicial fue respondido por 46 estudiantes presentes en el aula con un tiempo para las respuestas de 25 minutos. Se les pidió que identificaran el cuestionario con un código individual que nos serviría para correlacionarlos con los cuestionarios finales y mantener en esta recolección de datos el carácter anónimo. El cuestionario final fue cumplimentado, en los últimos 30 minutos de la última sesión de aplicación del CIMA. Contestaron el cuestionario más de 60 estudiantes, pero sólo hemos considerado para el análisis, las 38 respuestas en las que teníamos constancia, mediante la identificación por código, de que el mismo estudiante había realizado el cuestionario inicial y final.

Los resultados comparados para cada una de las preguntas/problemas planteados se recogen en las escaleras 
de aprendizaje que representamos en las figuras 3 a 6 . En cada escalón se recogen los tres modelos de respuesta, A, B y C. El aprendizaje se desarrolla desde los niveles más alejados al modelo de respuesta óptimo, en nuestro caso, c. Sobre cada escalón se sitúan los individuos cuyas respuestas se han incluido dentro de cada modelo, según la nomenclatura $X / Y$, siendo $X$, el número de estudiantes que han respondido según ese modelo en el cuestionario inicial y siendo $Y$, el número de alumnos/as que respondieron según el modelo en el cuestionario final. Bajo los escalones se detallan los modelos de respuesta así como los valores porcentuales que representan en las respuestas iniciales y finales sobre el total de 38 observaciones consideradas.

La progresión del aprendizaje en el primero de los problemas planteados (figura 3) muestra un desarrollo adecuado del aprendizaje culminado con un 73.7\% (28/38) de los estudiantes en el modelo óptimo de respuesta. Asimismo, como se observa en la tabla 1 el $70 \%$ de los estudiantes han mejorado en su nivel de respuesta. En la figura 4 , se observa para el segundo de los problemas planteados, un incremento importante en el ascenso desde el nivel básico al intermedio, si bien en el nivel óptimo de la escalera apenas se ubica un tercio de los estudiantes. El progreso del moldeo de respuesta al menos ha sido positivo en un $60 \%$ de los individuos observados.

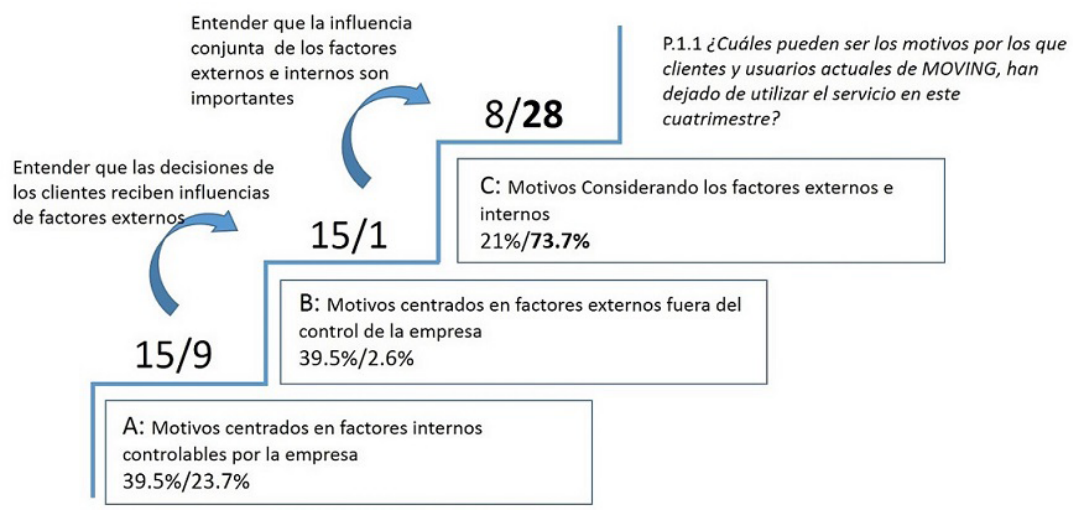

Figura 3. Escalera de aprendizaje P.1.1

Jornadas de Formación e Innovación Docente del Profesorado | № 2 (2019) Esta obra se distribuye con la licencia Creative Commons Reconocimiento-NoComercial-SinObraDerivada 
Con relación al tercero de los problemas, cuya escalera de aprendizaje representamos en la figura 5, observamos un comportamiento similar al anterior, en el que la mayoría de los estudiantes, aun mejorando con su aprendizaje, no han llegado al modelo de repuesta más elevado, en el que se han situado tan sólo el 23.7\% del grupo. La última de las preguntas (figura 6) analizadas sitúa a la mayoría de los estudiantes, el $52.6 \%$ en el modelo de respuesta óptimo y al resto en el modelo intermedio. Resultados muy valorables en cuanto que el obstáculo a saltar desde el escalón intermedio al superior conlleva un mayor desnivel.

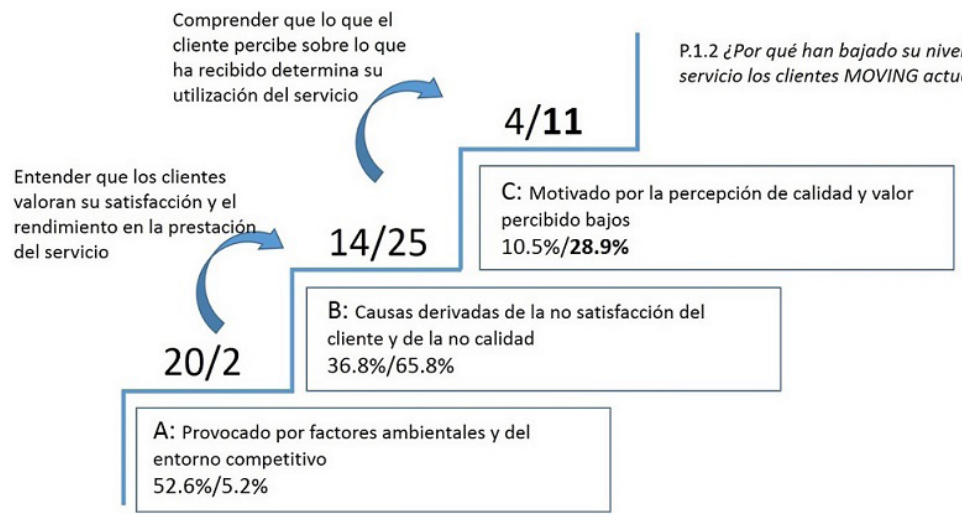

Figura 4. Escalera de aprendizaje P.1.2

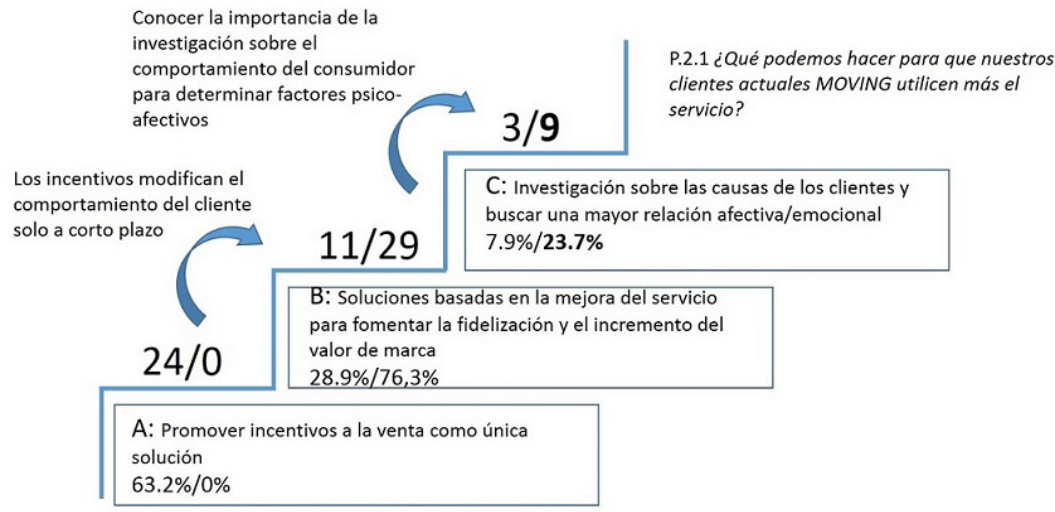

Figura 5. Escalera de aprendizaje P.2.1

Jornadas de Formación e Innovación Docente del Profesorado | № 2 (2019) Esta obra se distribuye con la licencia Creative Commons Reconocimiento-NoComercial-SinObraDerivada 
El valor del cliente para este servicio está en aspectos

relacionados con la sostenibilidad

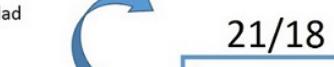

B: Acciones relacionadas con la sostenibilidad y el

$5 / 0$

A: Acciones no relacionadas con la sostenibilidad

$13.2 \% / 0 \%$

Figura 6. Escalera de aprendizaje P.2.2

Los niveles de desarrollo iniciales y finales del aprendizaje individual se detallan en la tabla 2. Las observaciones consideradas son la de los estudiantes identificados que han respondido a las cuatro preguntas (P.1.1, P.1.2, P.2.1, P.2.2) del cuestionario inicial y final. La columna de las diferencias (dif) denota con la numeración positiva o negativa el salto de niveles en la escalera de cada individuo para cada pregunta. 
Tabla 2. Niveles de desarrollo iniciales y finales

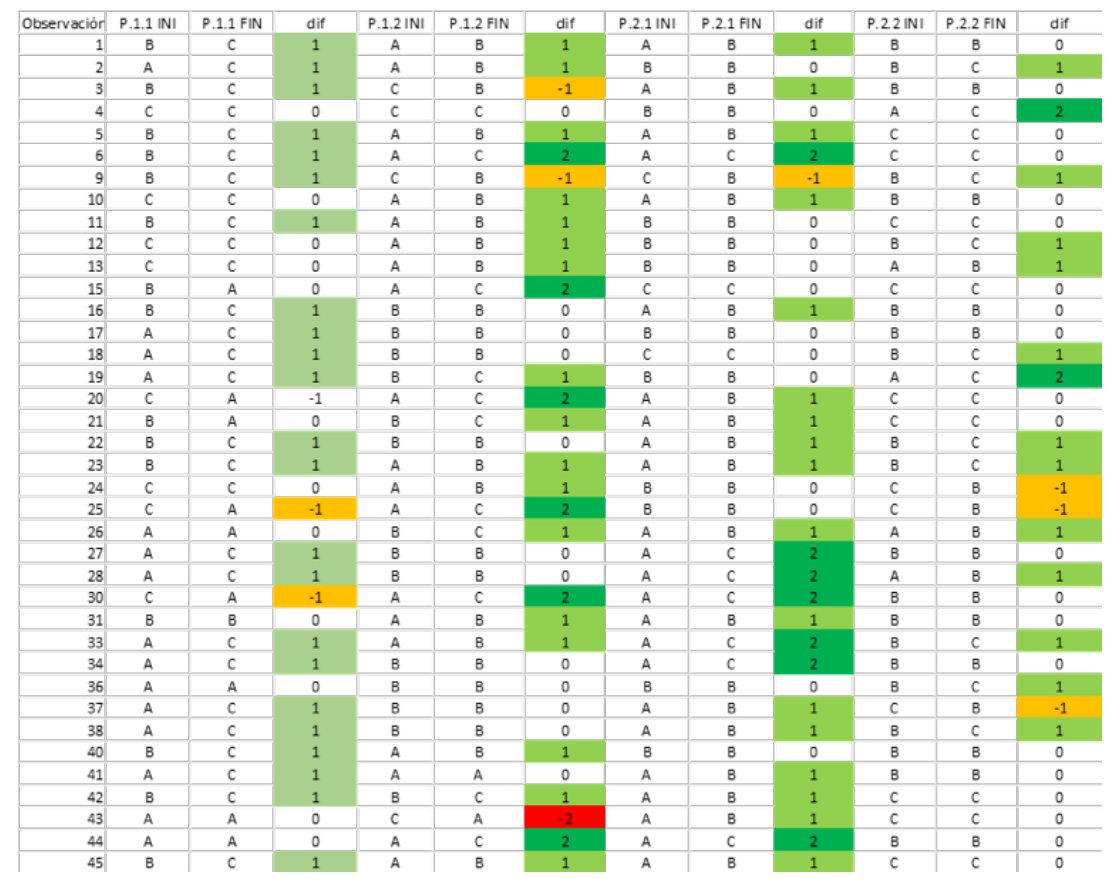

\section{Evaluación del CIMA}

\section{Análisis argumentado de la aplicación del CIMA}

\section{Lo que ha funcionado.}

En general la valoración y el saldo de la experiencia, positivos. Un nivel de participación alto, a pesar del elevado número de estudiantes en algunas sesiones, que no conseguíamos con los modelos metodológicos empleados con anterioridad. Los estudiantes han estado más activos y se ha podido mantener la atención y la participación durante casi todo el tiempo de las sesiones, más largas (10 minutos más) de lo que están habituados en la asignatura.

Jornadas de Formación e Innovación Docente del Profesorado | № 2 (2019) Esta obra se distribuye con la licencia Creative Commons Reconocimiento-NoComercial-SinObraDerivada 4.0 Internacional (CC BY-NC-ND 4.0.) 
Creo que el planteamiento del contexto inicial (director/a de marketing de una empresa de servicios) y las sucesivas preguntas encadenadas, han permitido que los estudiantes se pusiesen en una situación real profesional que les ha planteado tener que razonar, presentar y argumentar sus ideas en grupo y ante la clase. Los estudiantes se sienten cómodos cuando se les pide que participen y reflexionen, y a algunos les gusta el debate en clase y salir a la pizarra, por lo que si se motivan suficientemente creo que se puede sacar más provecho al modelo metodológico.

\section{Lo que no ha funcionado}

La formación de los grupos y el trabajo con la ficha del caso no ha sido bien planteada por el docente. Desde el principio, sabiendo que parte de la evaluación se sostendría sobre la actividad de los grupos recogidas en la ficha, debíamos haber planteado la formación de los grupos como un compromiso para seguir de forma consistente durante todo el proceso. La conexión con los estudiantes antes del inicio del proceso y durante su aplicación deberían haber sido suficiente, como así se demostró en la sesión última, para que los grupos tuviesen conciencia de que tenían que mantenerse durante todo el proceso.

Como consecuencia del mal funcionamiento de los grupos, el instrumento que debía servir para evaluar a actividad grupal, la ficha del caso, no ha cumplido con su misión, ya que me encuentro fichas cumplimentadas cada sesión por estudiantes diferentes. La ficha debería haberse proporcionado el primer día como un material impreso y a entregar al final del proceso tras el trabajo consistente de las sesiones por los grupos.

En cualquier caso, trabajar con grupos de estudiantes tan numerosos, por encima de 50, y en espacios fisicos tan poco adecuados para el trabajo en grupos, han supuesto 
un claro freno para que el clima de la clase fuese más participativo, sobre todo a la hora de poner en debate las opiniones de los grupos voluntarios o seleccionados que salían a exponer en la pizarra.

\section{Lo que se puede mejorar}

- Formación y gestión de los grupos. Dejar claro desde el inicio, la importancia del trabajo en equipo, la consistencia y el compromiso a exigir a los miembros de asistir a las sesiones y trabajar con la misma ficha, y los mismos compañeros, durante todo el proceso. Una vez formados los grupos con sus componentes, publicar en enseñanza virtual la composición de los mismos y la necesidad de trabajar cada sesión.

- Ficha del caso. La ficha debe ser impresa con un formato a modo de informe para que puedan ir cumplimentándola y entregar al final del ciclo. La ficha es el documento de evaluación de la actividad grupal y así deben saberlo ellos.

- Comunicación con los estudiantes entre sesiones. Establecer una comunicación entre sesiones, vía mensaje en la plataforma de enseñanza virtual, para instarlos a la reflexión sobre lo que se ha realizado en cada sesión y lo que realizaremos en la siguiente. Con ello logramos que se impliquen y participen más y se sientan llamados a la próxima sesión.

- Inclusión pregunta. Debemos incluir en el CIMA, una tercera pregunta, la P.2.3 que ha sido formulada para la conclusión del caso planteado y que no se incluyó en el mapa conceptual ni en el cuestionario inicial.

- Material para trabajo fuera del aula. Sería conveniente contar con material para trabajo y reflexión del estudiante fuera del aula. Material que debería trabajar y constatar su esfuerzo mediante fichas bibliográficas a entregar entre sesiones.

- Control de firma. La asistencia a las clases debe ser controlada, más allá del trabajo en equipo que 
hagan constar en la ficha del caso. La exigencia de un $80 \%$ de asistencia parece una buena manera de motivar la asistencia continuada de los estudiantes.

\section{Elaboración del Modelo Didáctico Personal a partir de la autoevaluación}

Como reflexión personal, en base a la experiencia vivida con el diseño y la aplicación del ciclo de mejora del aprendizaje aplicado en la asignatura de Marketing de Servicios, impartida en el cuarto curso del Grado en Marketing e Investigación de Mercados, exponemos, a continuación, unas breves ideas sobre el aprendizaje de los estudiantes, el contenido a enseñar, la metodología empleada y la evaluación del proceso de enseñanza-aprendizaje.

En cuanto al aprendizaje de los/as alumnos/as consideramos que hemos obtenido unos resultados aceptables, con una progresión, mostrada en el mayor nivel de las argumentaciones dadas en las actividades planteadas en el aula, confirmada después por los resultados del contraste de los modelos de respuesta entre el cuestionario inicial y final que se observa en las escaleras de aprendizaje. El papel más protagonista de los estudiantes en todo la aplicación del ciclo, creemos les ha servido para mostrar más interés en la resolución del caso central y una actuación mucho más participativa que en la que obteníamos con modelos más tradicionales.

En cuanto a los contenidos de enseñanza debemos reconocer que, pese a la dificultad inicial planteada por el diseño de los mapas conceptuales, han sido una herramienta muy eficaz para lograr modelar todo el contenido del tema alrededor de una situación cuasi real que les ha permitido a los estudiantes reflexionar e ir descubriéndolos de forma muy autónoma. El mapa y sus relaciones han sido fundamentales para poder diseñar la propuesta 
basada en problemas y encontrar las preguntas adecuadas para su desarrollo.

En relación con la metodología entendimos que los modelos tradicionales, basados en la comunicación a través de la transmisión de conocimientos (De Alba-Fernández y Porlán-Ariza, 2017), debían ser sustituidos por modelos más participativos, estimulantes para los estudiantes y capaces de lograr su motivación para ir desarrollando sus propias ideas y argumentos para dar respuesta a una serie de problemas estructurados a través de preguntas encadenadas. Por ello, no es solo cuestión de dar más participación al estudiante, sino hacerlos reflexionar para ir dando solución a problemas extraídos de contextos reales, que les vayan permitiendo descubrir los conceptos necesarios para lograr el aprendizaje esperado. Como herramienta fundamental para el éxito de la aplicación de este modelo, consideramos el diseño de la secuencia de actividades pieza clave para que el docente pierda su papel, eminentemente, protagonista para asumir un papel más de guía en el proceso de desarrollo de las propias ideas del estudiante.

Para comprender y estimar el éxito del proceso de enseñanza-aprendizaje debemos plantear la evaluación del mismo. La evaluación a través de las escaleras de aprendizaje, resultado del uso de los cuestionarios inicial y final, permite de forma simultánea la evaluación del proceso completo. Conocer la progresión del grupo a través de la escalera mide la eficacia en la trasmisión del conocimiento (cómo ha enseñado el docente), y el análisis de los resultados individualizados permite evaluar el aprendizaje (qué ha aprendido el estudiante).

Al ser un proceso secuenciado mediante actividades diseñadas y programadas en diferentes sesiones, permiten ir comprobando, con los resultados parciales obtenidos, qué modificaciones serán necesarias realizar, gracias 
al feedback obtenido de cada sesión. Con ello es posible, no sólo la mejora continua para diseños futuros, sino la modificación en tiempo real al ir midiendo los resultados entre sesiones recogidos en el diario de sesiones.

El conjunto de estas reflexiones conforman nuestros Principios Didácticos Personales y que sintetizamos en cuatro: 1) el papel protagonista del estudiante en el proceso de aprendizaje; 2) la organización de los contenidos a enseñar en mapas conceptuales; 3 ) el planteamiento de problemas a resolver por el estudiante; y 4) la evaluación continua planteada como una evaluación secuencial real. Estos principios nos servirán como esqueleto del Modelo Didáctico Personal de referencia.

Jornadas de Formación e Innovación Docente del Profesorado | № 2 (2019) Esta obra se distribuye con la licencia Creative Commons 


\section{Referencias bibliográficas}

BAIN, K. (2005). Lo que hacen los mejores profesores universitarios. Valencia: Publicaciones Universidad de Valencia.

DE ALBA-FERNÁNDEZ, N. y PORLÁN-ARIZA, R. (2017). La metodología de enseñanza. En R. Porlán (Coord.): Enseñanza universitaria. Cómo mejorarla, (pp. 37-54). Madrid: Morata.

FINKEL, D. (2008). Dar clases con la boca cerrada. Valencia: Publicaciones Universidad de Valencia.

MORA, F. (2017). Neuroeducación: Solo se puede aprender aquello que se ama. Madrid: Alianza Editorial.

PORLAN, R. Coord. (2017). Enseñanza universitaria. Cómo mejorarla. Madrid: Morata.

Jornadas de Formación e Innovación Docente del Profesorado | № 2 (2019) Esta obra se distribuye con la licencia Creative Commons 


\section{Notas al final}

1 Hemos empleado en todo momento un nombre ficticio (MOVING) para la empresa protagonista del caso práctico central del modelo de aprendizaje. Con ello, pretendemos evitar posibles conflictos con relación al uso de una marca registrada, pero el nombre elegido era claramente alusivo a la empresa real referida. 